alkoholischen Auflösung bleibt das schwofelsaure Glyoyrrhizin als ein dunkelbrauner durchsichtiger Körper zarück. In kuchendem Wasser ist es nach längerem Kochen löslich; die Auflösung ist vollkommen nentral.

Um die Mengo der Sohwefelsăure in diesem Salze zu bestimmen, löste ich eine durch das Gewicht bestimmte Quantität schwefelsauren Glycyrrhixins in Wasser auf und versetzte die klare Auflösang mit Chlorbaryumlösung, bis kein weisser Niederschlag mehr entstand. Da das Glycyrrhizin mit Baryt eino Verbindung eingeht, so wurde hierauf der Niedersablag mit reiner Salzsaiure in der Wärme behandelt, welche die Glycyrrhizin-Verbindang mit Baryt auflöst and den gebildeten schwefelsauren Baryt zurücklässt. Dieser wurde nan abfiltrirt, getrocknet, gewogen und der Gehalt an Schwefelsäure daraus berechnet. Es fand sich, dass das schwepelsaure Glycyrrhizio In $100 \mathrm{Th}$. aus 7,34 Schwefelsäare und 92,66 Glycyrrhizin besteht.

II.

\title{
Chemische Untersuchung des Guano Von
}

C. BFRTES in Regenwalde.

England ist vermöge seines ausgebreiteten Verkehrs za Wasser und zu Lande derjenige Siant, welcher sich am leichtesten aus der Nübe und Perne, sei es auch mit bedeutenden Kasten und Mühen verknüpft, ausgezeichnete Mittel zur Beför derung der Industrie und des Ackerboues verschafien kann. So hat England jäbrlich bedeutende Summen zum Aukauf von Kaochen nach Deutschland gesendet, am seinen Cultur-Flächen durch ein kräftiges Düngungsmaterial das zu ersetzea, was ihnen vielleicht schon seit mehreren Jahrhunderten darch einen zu starken Getreide-(besonders Weizen-) Bau entzogen wurde.

Da in Folge der vielen in Dentschland errichteten Rankelrüben-Zuackerfabriken, deren Bedarf an Knochen zar Zntfäbung sehr bedentend ist, der Preis derselben sehr stieg, stellte aich 
das Bedürfniss eines andern wohlfeilen Düngungsmaterials herans, und es kam ein englischer Schiffer aup die glückliche Idee, wegen Mangels an Rückfracht eine Ladung des bei den Peuanern zur Düngung sehr geschätaten und in ungeheuren Massen vorhandenen Gunno nnch Engiand mitæunehmen.

Die mit dem Guano angestellten chemischen Versuche zeigen, dass wir bis jelzt noch kein eituiges Düngungsmittel kennen, welches in einem so hleinen Volumen eine so grosse Menge von allen Pfancennuhrungstheilen enthlelle.

Das Guanopulver, welches ich einer chemischen Untersuchurg unterwarf, bokam ich durch dic Gefälligkcit des Herrn Oekonomic-Commissions-Ruths Spre ngel, der davon gwei Proben, eino blassgelbe und eine rothbraune, von Liverpool zugegefickt erbalton batte.

Um ein vallig gleiches Material verwerden zu künnen, gchätete ich meinen anzen Vorralh, etwa $1 / 4$ Pfil. hetragend, in eine Reibschale, rührle die Masse darin gut durch einander und benutzle dieselbe nun za den versehiedenen Operationen. Nach Winterfeld giebt es drei Arten des Guano, weissen, gelben und rothen; jch untersuchte den letotern, und dieser hatte folgende Eigenschafion. Das Guanopulver besilat eine duntel-hraunrothe Farbe, einige darunter befindiche, noch nicht gan\% zerfallene grössere Stücke zeigten inwendig noch eine weissgranc Farbe und ein blittriges Gerüge. Lnter dem Pulver befarden sich weder Federn noch sonstige regetabilische Reste, wie jch diese in grosser Monge in dem gelben Guanopulver gesehen habe. Das Pulver hatle einen ejgenthümlich stechenden, urinösen, aber doch bei weitem nicht so starken Greruch als der gelbe Guano. Auf nasses rothes Lakmuspapier gebracht, reagirte es stark ammoniakalisch, indem dis Reaction grosstentbeils an der Lurt wieder verschwand. Das trockne Pulver gab eine Menge Ammoniakgas aus, welcbes sich sehr leicht nacbweiken liess. Ich schüttete nïmlich elwas ouf eine Untertasse, atellie einen Giastrichter darüber und bing in die Oefnung deagelben cinen Streifen nasses rothes Lakmuspapier, welches schon nach einigen Minuten völlig wieder blan wurde. Auch brachite cin darüber gehaltenes, mit Salzsâure befeuchtetes Glasstäbchen eine beträchtliche Menge weisser Dämpfe hervor. 
Wenn 10 Gr. Guanopulver mit etwa $40-50$ Gr. Wasaer übergossen wurden, kühlte sich dieses Gemisch um $\mathbf{5}^{n} \mathbf{R}$. ab. Die Auflösung in kaltem Wasser reagirte stark ammoniakalisch, sie war fast wasserhell, dagegen die mil beisøem schwach gelb gefärbt.

Da ich mich durch die vorhergegangene qualitative Analyse belehrt hatte, welche Kürper in diesem Guanopulver vorkommen, so werde ich gleich zur quantitativen Bestimmung der darin befindlichen Körper übergehen.

lch habe schon früber erwähnt, dass ich mir durch sorgfältiges Zusammenmischen cin unter sich gleiches Material verschafft hatte, deshalb konnte ich unbeschadet der Genauigkeit zur Abscheidung der meisten Körper neue Portionen in Untersuchung nehmen.

\section{Bestimmung des Kochsalzes, des schwefelsauren Kali's und Natrons.}

Zur Bestimmung dieser Kürper wog ich 5,0 Gr. des Pulvers ab, glühto sie im Platinticgel und erhielt darnach 8,110 einer weissen porösen Asche. Beim Erhitzen rochen die Dämpfe eigenthümlich; stärker erhitzt, nahmen eie den Geruch an, wie wenn glühendes Eisen in Wasser abgelöscht wird. Mit Wasser ausgezogen, reagirte die Auflösung schwach alkalisch; sio wurde in 4 gleiche Theile getheilt und daraus bestimmt:

a) die Menge Schwefelsäure durch Barytaulösung und davon 0,093 Mgr. gefunden;

b) die Menge Chlor im Kochsalze durch Silberaunösung and davon 0,143 Mgr. gefunden;

c) die Menge Kali durch Platinaufoosung und davon 0,158 Mgr. gefunden;

d) diente als Reserve.

Berechnen wir aus dem schwefelsauren Baryt dto Menge Schwefelsäure $(2,529)$ und geben zuerst dem Kali 2,325 seinen entsprechenden Antheil 1,902, so crhalten wir 4,227 schwefeleaures Kali, and es bleibt uns nun noch ein Ueberschuss von $0,627 \mathrm{Mgr}$. Schwefelsăure. Da ich nun in dem Reserveviertel nur Spuren von Kalkerde und garkeine Talkerde gePunden babe, so mass obige Menge Schwefelsäure mit Natron verbunden darin vorkommen. Es werden die 0,627 Mgr. Schwefelsšurc 0,492 Mgr. 
Natron gebrauchen und damit 1,119 wasserfreies schwepelsadres Natron bilden, aber nicht als solches, sondern als wasserhaltiges darin entbalten sein. Wir haben von dicsem Wasserauszuge nun noch das Viertel zu betrachten, welches jar Bestimmung des Kochsalres gedient hat. Ich fand dario 0,143 Mgr. scharf getrocknetes Chlorsilber, oder in 100: 11,440. Nehmen wir an, dass 100 Chlorsilber aus 25 p. C. Chlor und 76 Silber bestehen, so würlen dic 11,440; 2,860 Chlor enthalten und diese 1,879 Narrium zu Kochsalz gebrauchen $=4,739$.

Bei einer andern Gelegenbeit, wo ich zuerst die Mengo Salmiak durch Ausziehen mit Alkohol entfernte und dann erst den in Alkohol ungelöst geblicbenen Rückstand gluhte, darauf mit Wasser auszog und diesen Auszug mit den 21 Mgr. betragenden Salzen vermischte, die ich nach Zerstörung des Salmiaks durch Feuer erbiclt, und nun aus dieser ganzen Flüssigkeit das vorhandene Kochsalz, wie oben angegeben, bestimmle, betrug die gefundene Menge an Chlorsilber in $\mathbf{5}, \mathbf{0}$ Gr. deg Guanopulvers $12 \mathrm{Mgr}$, oder in 100:0,240 dividirt durch $4=60$ Chlor und 40 Natrium zu 0,100 Kochsaly, und scheint elne grüssare Menge in diesem nicht vorzukommen.

Um mir darüber völligo Gewisshcit zu verschaffen, habe jch beide Versuche anf Jieselbe Weise noch einmal wiederholt und fast gan\% genau dieselben Resultate erhalten. Es muss demnach beim Glülien ein anderes Natronsaliz so zersetzt sein, dass scine Säure mit ejner anlern Base, and die Base oder das Radical der Base mit einem negaliven Stoffe (hier mit dem Chlor des Salmiaks) in Verbiniung getreten sein, und gleichzeilig feucrbeständiges Kochsalz gebililet haben. Die Sïure des zersetzten Natronsalzes kann wohl keine andere als Phosphorä̈ure gewesen scin, die sich nach Zersiörung der Oxalsäure des im Guanopulver vorkommenden oxalsuuren Kalkes mit lot\%terer verbunden hat, während das frei werdende Natron, jolat Natrium, seinen Antheil Chlor aus dem Salmiak fest hielt, und so dio oben angegebene grossere Menge Kochsalz entstand.

\section{Beslimmung des phosphorsauren Natrons.}

Ans diesen so eben beschriebenen Versuchéd lässt sich wobl mit Bestimmiheit die zersetzte Menge des vorhanden gewesenen phosphorsauren Natrons ableiten ano dem Gewicte 
nach bestimmen, wenn wir dle Menge Natrium aus dem Ueberschusso vom gefundenen Kochsaly eu Natron und dieses mit Phosphoraäure su phosphoraaurem Natron berechnen.

In der eigentlichen Menge des vorbandenen Rochaslzes sind 0,040 Natrium enthalten; ziehen wir diese von der oben gefundenen Menge 1,879 ab, so bleiben 1,839; diese gebrauchen 0,632 Sauerstoff und bilden damit 2,471 Narron, und dieses wird, um einfach-phosphorsaures Natron zu bilden, 2,820 Phosphorsäure gebrauchen. In 100 Gewichtstheilen dieses Ganopat. vers werden demnach 5,291 dieses Satzes vorbanden zein. Denn es lässt sich wohl nicht annebmen, dass, da noch eine bedeutende Menge Chlor aus dem Salmiak vorbanden war, dieces eine andere Menge noch vorhandenes pbosphersaures Natron unkersetzt gelassen hätte.

Bestimmung des Salmiaks, so wie einer wachs - und har:artigen Materie.

Un die Menge Salmiak abzuscheiden, nahm ich 6,0 Gr. des lufurocknen Guanopulvers in Untersuchung und $20 \mathrm{~g}$ diese in gelinder Wärme mit 94 p. C. Alkohol aus. Der alkoholische Auszug: war gelb gefärbt und gab, bei sehr gelinder Wirme bis zur Krystallisation abgedampft, einen salzigen Rückstand von 0,376 Mgr. Die Salzo in Wasser gelüst, die Auftösung decantirt, dns Ungelösto getracknet, erhielt ich 0,030 Mgr. eines wachs- und barzähulichen Körpers, oler in 100 Gewichlstheilen 0,600 dieser Materic. Die decantirte wassrige Auflösung, zur Trockenheit verdampft und dann schwach geglihh, ergab einen salzigen Rückstand von 0,021 Mgr. (wovon frtber beim Kochsalze die Rede war). Zielien wir die 51 Mgr. von dem obigen ganxen Gewichte $0,376-51$ ab, so bleißen 0,325 Mgr. für Salmiak übrig, welches, aup 100 berechnet, 6,500 beträgt. Vielleicht war er noch mit einer nicht năher untersucbten organischen Materio vermischt, aber ulcht mit Harnatoff, der nicht mehr darin za finden war.

Die 21 Mgr., die nach dem Glühen des Salmiaks übrig geblieben waren, bestanden aus einer geringen Menge Kochsalz, Kalk- und Talkerde. 


\section{Bestimmung des oxalsauren Ammoniaks.}

Nach dem Alkohol wandte ich bei einer andern gleichen Menge kaltes and heisses Wasser zam Augziehen des Pulvers sn. In dicser Aurlösung flnden sich nach der qualitativen Untersuchung oxalsaures Ammoniak, barneaures Ammoniak, phosphorsaures Natron (phosphorsaures Natron-Ammoniak ?), schwefelsaures Natron, schwefelgaures Kali, phosphorsaure Ammoniak-Talkerde und oin Theil Kocbsaly. Wir haben die Menge einiger daraus schon kennen gclernt und wollen noch die übrigen $\mathrm{zu}$ bestimmen suchen.

Um das Gewicht vom vorhandenen oxalsauren Ammoniak zu bestimmen, setzle ich der Hälfle dieser wassrigen Auflösung so lange in Wnsser gelösten Gips zu, als dadurch noch eine Trübung erfolgte. Der Nlederschlag, aus kleesaurer Kalkerde bestebend, durch ein gewogenes Filter flltrirt, mitheissem Wasser susgewaschen, scharf getrocknet und gewogen, ergab $0,453 \mathrm{Mgr}$. oxalsauren Kalk oder, nut 100 des Guanupulvers berechnet, $0,453 \times 2=0,906 \times 2=1812 \times 10=18,120$; diese entsprechen in $100=44$ reiner Kalkerle und 56 Oxalsäure. Die $18,120 \mathrm{Th}$, enthalten 10,147 Oxalsäure, and diese gebrauchen, um einfach-oxalsaures Ammoniak zu bilden, $76: 24=10,147$ : $x=3,204$ Ammoniak und geben damit 13,351 oxalsaures Ammoniak in 100.

Bestimmung des Harnstoffes und harnsauren Ammoniaks.

Das harnsaure Ammoniak habe ich aus einer neuen gleichen Portion bestimmt. Sle wurde zu wiederholten Malen mit erwärmtem Wasser anggezogen. Die letzlen Auszüge wurden zuerst verilampft and dann die ersten zagesetzt und nun bis zur Honigconsistenz eingedamprt. Um diesen Auszug auch zugleich auf Harnstof zu untersachen, brachte ich die Masse in ein vierfaches Volumen 94 p. C. Alkohol, liess das Gemisch elnige Stunden zugederkt stehen, flltrirte die weingeistige Auflösang ab, lampfte sie bei gelinder Whirme bis auf $1 / 4$ ein, liess das Gemisch erkalten und setzte es dann in mit wenig Wasser vermischtes Gonnopulver and kühlte dasselbe darin nooh mehr nb; non wurden nach und nach $11 / 2$ Vol. Salpetersiare sugegossen and das Gemisch in einer neuen Menge mit 
Wasser abergossenen Guanopoivers zam allmahligen Abkühlen noch cinige Zeit stehen gelassen. Fs hatte slcb nber nach mehreren Stunden kein salpctersaurer Harnstof ahgeschieden, mithin war keiner vorhanden.

Nach Auswaschen des Rückstandes mit Alkohol übergoss ich denselben mit ziemlich concentrirter Salzsaiure; das dadurch gefällte, etwas gelb gefărble körnige Pulver wurdo auf cin gewogenes Filter gebracht und darin so lange mit kaltem Wasser ausgewaschen, bis keine Salzstiure mehr vorhanden war. Das Pulver, bei etwa $80^{\circ}$ R. getrocknet und gewogen, ergab das Gewicht von 0,146 Mgr., vielleicht nocb mit geringen Mengen Harnblasenschleim verunreinigt; 5 ; 0,146 , mithin in $100=2,920$ Harnsĩure, und diese gebrnuchen auf dieso Menge 0,321 Ammoniak und geben damit 3,214 harnseures $A$ amoniak.

\section{Bestimmung der phosphorsauren Ammoniak-Talkerde.}

Um die quantitative Menge dieses Tripolealzes zu bestimmen, nahm ich wieder 5,0 Gr. Guanopulver in Arbeit, zersiorte zuerst alle verbrennlichen Körper, übergoss die Asche mit Wasser, erwärmte damit einigo Zeit und setzte nun so lange 8alysüure zu, als sich darin noch elwas aufzuläsen schien und die Flussigkeit stark sauer reagirte; sie wurde durcb ein ungewogenes Filter gegossen, erkaltet und non bis zur alkalischen Renction mit Ammoniak versetat, der erhaltene Niederschlag von phosphorsaurer Kalkerde abfihrirt, mit helseem Wasser ausgewaschen, sonst aber weiter nicht benulyt, da seine Menge, nacb dem Frühern, nuf Jiese Weise nicht mit Genavigkeit beetimmt werden kann. Kben so konnte auch der Niederschlag von Kalkerde nicht benutzt werden, da auch diese Menge die unrichtige sein muss.

Bie von der oxalsauren Kalkerde abfiltrirte Flässigkeit konnle ich nun erst zor Prüfung aup Talkerde benutzen. Ich versetzte sie mit einer hinreichenden Menge von phosphorsaurem Natron und einem Deberschusse von Ammoniak, rührte die Flüssigkeit gut durch einander und liess sie, bedeckt, 12 Stunden steben, filtrirte dann dorch ein gewogenes Filter, süsete den Niederschlag mit ammoniakhaltigem Wasser aus, trockneto schart, so dass Wasser und Ammouiak entwicben seiu konnted, 
und erhlelt dann 0,161 Mgr. phosphorsaure Talkerde, oder in 100 Gewichtstheilen Gumnopalver 4,196 halb-phosphersaure Ammoniak-Talkerde.

\section{Bestimmung der phosphorsauren Kalkerde, der Alaunerde} und des in Salpetersäure unlösliclten Kückstandes.

Naehdem 5,0 Gr. Guanopalver mit Alkohol and Wasser vorher ausgezogen waren, wurde der hiernach erhaltene Rückstand 1,940 gegläht, und dieser liess darnach 1,542 Mgr. Asche. Sie wurte so lange mit diluirter Salpetersäuro behandelt, als diese Säure noch etwas auflöste, die stark sauer reagirende Auflosung erbitzt, dann durch oin gewogenes Filter flltirt, damit die mineralischen, nicht anfgelösten Theile zurückblieben und sich nach dem Gewichte bestimmen liessen. In diesen 5,0 Gr. wurden 0,290 Mgr., oder in $100=5,800$ gefunden.

Die Auffösung in Salpetersïure wurde mit Wasser verdünnt und dann bis zur stark alkalischea Reaction mit Ammoniak-Flüsigkeit versetzt und der dadureh bewirkte Niederschiag von phosphorsaurer Kalkerde und Alaunerde sogleich durcb oin ungewogenes Filter filtrirt und während dieser Zeit sowohl das Filter als der Cylinder mit einer Glasplatte bedeckt, damit die Kohlenäure der Luft möglichst ubgebalten werde, indom sonst auch kobleneaurer Kalk mit abgesohieden wird. Nachdem der Niedersohlag auf dem Filter geammelt war, wurde derselbe mit heissem Wasser ansgewachen, das Filter dann mit seinem Inhalte auf mehrfach zusammengeschlagenes Fliesspapief gelegt und der Niederschlag mit eivem Knachenmesser in eine Poreeltanschale gebracht, um die noeh Jaboi befindlicho Alaunerde in Auflosung zu bringen. Diess bewirkle ich durch Zusatz eines Ueberschusses von kohlensäurefreien Kali. Der in Kali völlig unlöslich gebliebene phosphorsaure Kalk wurde cogleich durch ein gewogenes Filter filtrirt, ausgewasehen, getrocknet und geglüht; es warden davon $0,497 \mathrm{Mgr}$. eihaliẹo, oder in $100=9,040$.

Iit dez durchfltrirten Flüssigkeit befindet sioh dis Alaunerdo, Un dieselbo abzucheiden, wachio ich die Flüsaigkeit dusch Balzsäure saver, orhitzte sio and setzte cinen kleinen Ueberschogs von kobleneanrem Ammoniak hinzu. Die Alaunerde 
scheidet stch dabei als Alaunerdehydrat in Flocken ab; sie wurte durch ein gewogenes Filter filtrirt, susgewaschen, getrocknet and gewogen und davon in 5,0 Gr. 0,008 Mgr. oder in 100 $=0,104$ gefunden.

\section{Bestimmung der oxalsauren Kalkerde,}

Aus der Flüsalgkeit, die nach Abscheidung der phosphorsauren Kalkerde and Alannerde durch Ammoniak and Abaltriren erbalten wurde, konnte ich nun die Menge Kalkerde, dic als oxalsaure Kalketde im Guanopulver vorkommt, genau abscheilen. Dieses geschah mit ciner Auflösnng yon oxalsnurem Ammoniak, so lange dadarcb noch eine Trübung exfolgte. Der Niederschlag, durch ein gewogenes Filter gegossen, mit beissen Wasser ansgewaschen, getrocknet und gewogen, und die so erhaltene Menge oxalsaure Kalkerile als die fo Ganopulver vorkommende angenommen, ergab in 5,0 Gr. 0.818 ; ditoss be. tràgt in $100=16,360$.

\section{Bextimmung des phosphorsauren Ammoniaks.}

Nachdem ith nun fast alle die in dfesem Guanopulver vorkommenden Körper auggeschieden and dem Gewichte nach bestimmt batte, blieb mir nur noch die quantitative Abscheldung des phosphorsauren Ammoniaks übrig, und diese machte hier einige Schwierigkeiten.

Um die Menge desselben zu bestimmen, zog jch $5,0 \mathrm{Gr}$. mit etwas erwirmtem Wasser aus, dampfte die Aurlysung ein und glühte den Rückstand, um alle verbrennlichen Körper weg zu schaffen. Nach dem Glühen wurde eine im Plass gewesene weisso Salmmasee erhalten die nach Abkobulang des Platintiegels in vi ele kleine Stuck zersprang. Mit Wasser ahergossen und erwürmt, löste sie sich vollstandig zn elner wasserhellen Flüssigkelt anf, die satuer rengirte. Um die durch Glühen entstandenen pyrophosphorsauren Salze in die gewöhalichen nmzuwandeln, erhitzte ich dieselben mit Wasser und Salpetersäure, dampfte sie zur Trockenheit ein, löste sie wiedex in Wasser anf und versetzte die Auflösung nun so lange mit ejner nantralen sa!pctersauren Kalknuflösung ưd einem kleinen Uabersebusze von Armoniak, ais dadareb noch oin Niederschlag von phogphor-

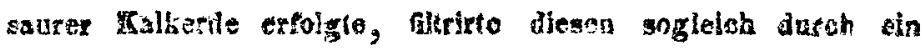




\section{Bertels, chem. Untersuchung des Guano.}

gowogenes Filler, slisste dasselbe mit heissem $W u s s e r$ aus, tracknete den Niederschlag und glühte ihn; es wurlen auf diese Weise in 5,0 Gr. 0,697 Mgr. 2/3 phosphorsaure Kalkerile gefunden.

5,0 Gr. gaben 0,697, mithin $100=13,940$, and diese enthalten 7,443 Phosplorsäure. Ziehen wir von dieser Menge die Phosphorsüure des phosphorsauren Natrons $\mathbf{2 , 8 2 0}$ ub, so bleiben noch $4,623 \mathrm{Mgr}$. übrig. Wir haben nun auch noch die Menge Phosphoraïure abzurechnen, die als phosphorsaure AmmoniakTalkerde lurch Wasser in Auflosung gekommen ist, nämlich

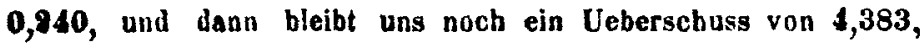
dis $\mathbf{8 , 0 6 7}$ Mgr. Ammoniak zu ihrer Sätigung gehrauchen. Es kommen demnach 6,450 phosphorsaures Ammoniak in diesem Guanopulver vor.

Bei einer trocknen Destillation, dic ich mit 2,500 Gr. des Palvers vornabm, erbielt ich $\mathbf{1 , 4 1 9}$ krystallisirten Salmiak, oder in $100=56,760$. Diese entbalten 18,163 Ammoniat.

Hieruach würde der von $\mathrm{mir}$ in Untersuchung genommene rothbraune luftrockene Guano in 100,000 Pfl. bestehen aus:

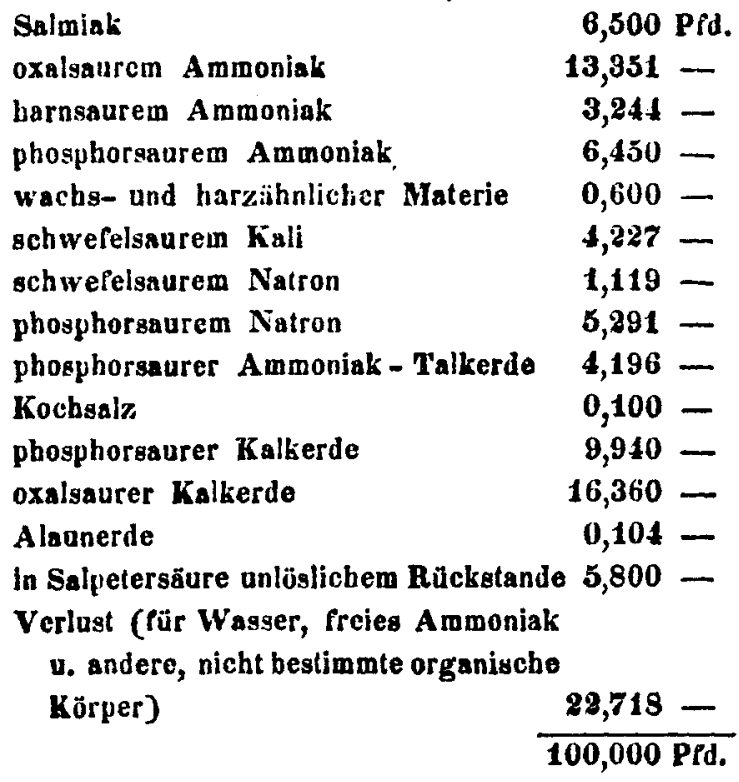

In den mir vorliegenden Resoltaten von $K$ la proth über das Guanopulver finde ich den Kochsalzgehalt mit 0,5 aufgembrt; in der sonst sehr ausfahrlich bearbeiteten Analgse von 
Pourcroy und Vaquelin wird gar kein Kochsalz angegeben, dagegen sind aber 4,2 Salmiak gefunden. Auf phospliorsaures Natron scheinen beide Herren entweder gar nicht nntersucht zu haben, oder sie baben keins darin gefunden. In 2 anderen, zwar nicht vollständig auggefübrten Analysen vom Prot. Johnston wird bei der einen der Gelalt an Kochsale, mit etwas phosphoraaurem Natron vermischt, zu 30,3 p.C. und bei einer andern, mit elwas schwelelsaurem und pbosphorsaurem Natron verwiscbt, zu 11,4 p.C. angegebeu. A as den Verauchen selbst geht hervor, dass Johngto den Kochsalzgehalt so wie den phosphorsauren Kalkgehult aus dem vorher geglübten Guanopulver bestimmt hat.

Schliesslich wandte ich dem in Salpetersäure unlüslichen mineralischen Rückstande nun noch meiue Aufmerksamkeit zu.

Ich wog 10,0 Gr. des Pulvers ab und schlïmmte sie. Ans dem erhaltenen Rückstande $\mathrm{zog}$ ich mittebt eines Magnetstahles 0,084 Mgr. Magneteisen ans (oder in 100=0,240). Da dem darch Scblämmen erhaltenen Pulver noch einige andere, vicht mineralische Korper belgemiacht geblieben waren, so zerstorte Jch diese durch Glühhitze, zog den hiernnch bleibenden Rückstand mit dllulrter Salzsäure aus und konnte nun die ungelöst gebllebeden Mineralien um so besser erkennen; sle bestanden grösotenthells aws grossen Quarzkörnern, schwarzem eisenbaltigem Mineral, mit wenig Foldspath and ziemlich viel Glimmerblistlchon vermigcht.

Die Auflösung in Salzabure liabo ich weiter nicht berdickAlchtigt.

Nachschritl. Seit den Analysen von Klaproth (Beiträge IV. 301) ist der Giane auth van $V$ ölckel analysirt worden (Ann. der Pharm. XXXVIL. 205). Die Resaltate belder Analysen wollen wir hior noch hinzurugen:

Aarnsuares Ammoniak

ozaleaures Ammoniak

oxalsanrer Kalk

phosphoreaures Ammoniak

phosphorsaures Ammoniak-Talt
Völckel. Klaproth.

$$
9,0 \quad 16,0
$$

10,6

$7,0 \quad 18,76$

6,0

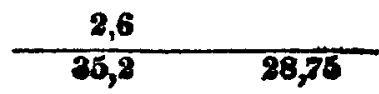


16 Ueb. ein neues Verfabren, reducirt. Indigo darzastellen.

\section{schwefelsaures Kali \\ schwefelsaures Natron \\ Salmiak}

phosphorsaure Kalkerde
Volckel. Klaproth.

Transport

35,2

5,5

3,8 Kochsalz 0,5

4,2

14,3

10,0

4,7

32,0

unbestimmte organische Materien, wovon

ungetühr 12 p.C. in Wasser unlöslich ; $32,3 \quad 28,76$

lösi, Kisensalx in kleinen Mengen, Wasser

$\begin{array}{ll}100,0 & 100,00 . \\ & \text { D. Red. }\end{array}$

III.

Ueber ein neues Verfahren, reducirten Indigo darzustellen.

(Auszug ans einem Briefe d. Hra. Fritzsc be an Hra. Chovre U1.)

(Compt. rend. T, X.T. p. 7ss.)

Meine Versbche aber den Indigo haben mich zu einer Methode, reines Indigblau darzustellen, gefübrt, welche ish deswegen um so mehr der Aufmerksnmkeit der Chemiker werth erachte, weil sle das Indigblau in krystallinischer Form liefert und weil sie eben so leicht als achnell auszuführen ist. Schon selt langer Zeit halte ich bemerkt, dass der Indigo, wenn man ihn mit einer alkoholischen Auplüsung von Kali behandelt, unter gewissen Umständen kieine Quantitäten von Indigblau in Blatteben absondert, and inden ich den Versueb wiederholte, gelang es mir, unfehthar immer dasselbe Resultat bervorrubringen. Es ist eine unfache Reduction des Indiga's, bei der man statt des Wassers Alkobol, und da die Substanzen, wciche man gewöbnlich zur Reduction des Indigo's anwendet, in Alkohol nicht löslich sind, stall derselben Traubenzucker anwendet; aus demselben Grunile muss man statt des kaustiseben Kalkes haustisches Kall oder Natron nehmen; das Neue der Methode bestebt dater vorzitglich in der Anwendung des Alkobnls, intem man irüher schon allo anderen Staffe, den Alkohol ausgenomxasn, angewand hat. 